\title{
Reflexión sobre la implementación de políticas etnoeducativas en la formación de docentes de matemáticas de la UDFJC ${ }^{1}$ \\ Reflection on ethnic forms of education policy implementation in the training of teachers of mathematics at the UDFJC
}

\section{A reflexão sobre as formas étnicas de implementação da política de educação na formação de professores de matemática na UDFJC}

Recibido: mayo de 2013

Aceptado: agosto de 2013
Ángel David León Sánchez ${ }^{2}$

Brianna Lorena Díaz Barreto ${ }^{3}$

Julián Daniel Sánchez Rincón ${ }^{4}$

Liceth Andrea Casallas Hernández ${ }^{5}$

\section{Resumen}

Este trabajo surge a partir de diversas discusiones que se plantean en un espacio de formación de docentes de matemáticas dentro de la universidad Distrital Francisco José de caldas, al ver el desinterés de $\mathrm{EPP}^{6}$ sobre la educación para poblaciones étnicas, por lo cual se intenta realizar una búsqueda de cómo en el transcurso del tiempo se fueron implementando políticas educativas que permitieran una conservación de la cultura de dichas comunidades, por tanto, se pretende identificar la manera en que se implementan dichas políticas en un espacio de formación docente como lo es la Licenciatura en educación básica con énfasis en matemáticas LEBEM $^{7}$

Palabras clave: Etnomatemática; políticas; etnoeducación; alumno; diversidad; cultura-religión..

\begin{abstract}
This work arises from various discussions that arise in an area of mathematics teacher education within the university Francisco José de Caldas, seeing the lack of PPE on education for ethnic populations, for which the user tries to how to search in the course of time were implemented educational policies that allow preservation of the culture of these communities, therefore, is to identify how these policies are implemented in teacher
\end{abstract}

1 Artíulo de Investigación

2 UD Francisco José De Caldas. Contacto: millosad10@hotmail.com

3 UD Francisco José De Caldas. Contacto: lore-2820@hotmail.com

4 UD Francisco José De Caldas. Contacto: julius9210@hotmail.com

5 UD Francisco José De Caldas. Contacto: andreita--11414@hotmail.com

6 Estudiante para profesor

7 Licenciatura en educación básica con énfasis en matemáticas 
education space such as the Bachelor of basic education with emphasis on math LEBEM.

Keywords: Ethnomathematics, political, ethnic education, student, diversity, culture, religion.

\section{Resumo}

Este trabalho surge a partir de várias discussões que surgem em uma área de formação de professores de matemática dentro da universidade Francisco José de Caldas, vendo a falta de EPP1 sobre educação para populações étnicas, por isso é tentada como pesquisar no decorrer do tempo, foram implementadas políticas educacionais que permitam a preservação da cultura destas comunidades, portanto, é identificar como essas políticas são implementadas no espaço de formação de professores, como o Bacharelado em educação básica com ênfase em matemática LEBEM2.

Palavras-chave: Etnomatemática, política, educação étnica, estudante, diversidade, cultura, religião.

\section{Introducción}

Un país como Colombia considerado territorio multicultural y plurilingüe según la constitución de 1991, ya que el 1.8\% de la población es indígena. Antes de la implementación de dicha constitución, la iglesia y el estado estaban encargados de educar y cristianizar las comunidades étnicas, imponiendo sistemas educativos con estructuras ideológicas y culturales ajenas a la concepción educativa dichas comunidades, siendo evidente el uso de la educación como mecanismo de desintegración cultural y asimilación de conocimientos ajenos a las necesidades reales de las comunidades indígenas, lo que generó desculturización y exterminio de la cultura, acabando así con comunidades como los: Ikas, los Kogui, y los Wiwas de la sierra nevada de Santa Marta, entre otras.

Son las comunidades indígenas las primeras en luchar por una educación que responda verdaderamente a sus necesidades, respetando y protegiendo su cultura, por tanto, se han venido generando políticas con el fin de propiciar el reconocimiento de estas comunidades, se reconoció que la mejor manera de proporcionar conocimientos que no a-culturicen la población, es formar como maestros a personas pertenecientes a la cultura, partiendo que los que más conocen de las comunidades indígenas son los indígenas.

Por último, se ven problemáticas a nivel educativo, generadas principalmente por la falta de docentes especializados, programas de capacitación y mecanismos de control inadecuados para la enseñanza en grupos étnicos del país, principalmente indígenas y afrodecendientes, a pesar que se estipulan en las diferentes leyes.

\section{Marco de referencia}

Politicas de Etnoeducación. En nuestro país Colombia, el gobierno se ve en la obligación de crear diferentes decretos y leyes que involucren tanto integridad como los derechos de las comunidades étnicas, generando las diferentes políticas etnoculturales, en estas se ven involucrados derechos como la vivienda, la alimentación, la salud y la educación. Para la elaboración de este trabajo se tienen en cuenta las diferentes políticas de etnoeducación, que tienen como objetivo posicionar una educación intercultural. Estás políticas educativas buscan que en todas las escuelas y colegios del sector oficial y privado del país entiendan que las culturas afro colombiana, indígena, gitana, entre 
otras, hacen parte de las raíces colombianas, avanzando así a una interculturalidad, es decir, hacer que en las escuelas se reconozcan y respeten las diferentes culturas.

Por tanto, dentro de las leyes que se han llevado a cabo en la constitución política de Colombia de 1991, en algunos de sus artículos se reconoce a Colombia como un estado pluralista, en el cual éste garantiza la protección de todos los habitantes. El castellano es el idioma oficial de este país, pero las lenguas y dialectos de los grupos étnicos son también oficiales en sus territorios. Se tendrá en cuenta la enseñanza que se imparta en las comunidades con tradiciones lingüísticas propias, en donde el castellano será la segunda lengua (art 1, art 2, art 10). Como lo menciona el artículo 68 "Los integrantes de los grupos étnicos tendrán derecho a una formación que respete y desarrolle su identidad cultural".

Así como la ley 70 de 1993, la cual tiene como propósito establecer mecanismos para la protección de la identidad cultural y los derechos de las comunidades negras de Colombia como grupo étnico, por tanto en unos de sus artículos referidos a etnoducación el art 32, "el estado colombiano reconoce y garantiza a las comunidades negras el derecho a un proceso educativo acorde a sus necesidades y aspiraciones etnoculturales".

Otro de las leyes que busca el respeto y así mismo la no desculturización de estas comunidades es el Decreto 804 de 1995 (Mayo 18) de la Ley 115 de 1994, "por medio del cual reglamentará la atención educativa para grupos étnicos", que la ley 115 de 1994 establece que la educación es un proceso de formación para los grupos que integran la nacionalidad, con estrategias pedagógicas acordes a su cultura, su lengua, sus tradiciones y sus fueros propios y autóctonos.

Etnoeducación - etnomatemática. El termino etnoeducación Desde el decreto Decreto 804 de 1995, se define como el derecho de los grupos étnicos "a una formación que respete y desarrolle su identidad cultural", y que se determina que ésta hace parte del servicio educativo, (Castillo, 2008).
La etnoeducación debe ser un medio de entendimiento entre culturas del país, a su vez servir como pretexto y herramienta para formar conciencia en los ciudadanos Colombianos. Cuando nos referimos a etnoeducación nos referimos a las prácticas educativas que tienen en cuenta procesos de comunicación, vida y desarrollos históricos de determinada cultural para impartir cualquier área del conocimiento.

Dentro de todas las áreas es pertinente hablar de etnoeducacion, como futuros docentes de matemáticas es importante conocer y hablar sobre la Etnomatemática, la definición de está es muy amplia, se puede hablar de ella desde la concepción que tenga el autor, por ejemplo una de las definiciones más notables es la del profesor Bill Bartón "las diferentes formas de matemáticas que son propias de los grupos culturales la llamamos etnomatemáticas" (Pacheco 2007), por otro lado Ubiritan D'ambrosio, para definir el concepto etnomatemática hace una composición de tres raíces etno, mathema y thica explicándolas de este modo (Blanco, 2008)

- Etno: entorno natural y cultural

- Mathema: artes, técnicas, maneras, estilos

- Thica: se refiere a la metodología y la forma de transmitir un nuevo conocimiento

Esquematizando estás tres raíces, Ubiritan D 'ambrosio llega a la siguiente definición, la etnomatemática, "sería las artes, técnicas de explicar, de entender, lidiar con el ambiente social, cultural y natural." (Blanco, 2008)

Por tanto, al recoger las definiciones dadas por dichos autores, se concluye que al referirse a etnomatemática se puede hablar de cualquier conjunto de personas que tienen una propia forma de comprender las matemáticas.

Para trabajar en el campo de la etnomatemática se debe reconocer que la matemática es una actividad humana que pertenece a la cultura, y que así como diferentes culturas tienen diferentes estructuras sociales y lenguas, tienen distintas matemáticas y 
como enfrentan distintos problemas en sus particulares entornos, generan distintas soluciones a los mismos, por lo tanto la matemática no se debe interpretar de una única manera.

Políticas de etnoeducación en un proyecto curricular que forma docentes en matemáticas

En la Facultad de Ciencias y Educación de la Universidad Distrital Francisco José de Caldas, la facultad de educación más grande del país, se evidencia que en el plan de estudios para los estudiantes solo se desarrolla una cátedra para toda la comunidad académica, dada a nivel general y por cumplimiento del artículo 39 de la ley 70 de 1993.

Por otra parte se evidencia que los docentes y estudiantes están interesados en realizar trabajos en el área de la etnomatemática y reconocen la importancia de educar en la diversidad, es decir, educar atendiendo a las necesidades de una comunidad.

Aunque desde el currículo del proyecto LEBEM no se vea a profundidad estudios sobre la etnomatemática, tanto los estudiantes como profesores buscan espacios en los que se pueda trabajar e investigar sobre la etnoeducacion, es por ello

Grafica 1:

\section{Plan de Estudios}

NÚCLEOS PROBLÉMICOS: ¿Qué es? ¿Cómo ha sido? ¿Cómo puede ser?
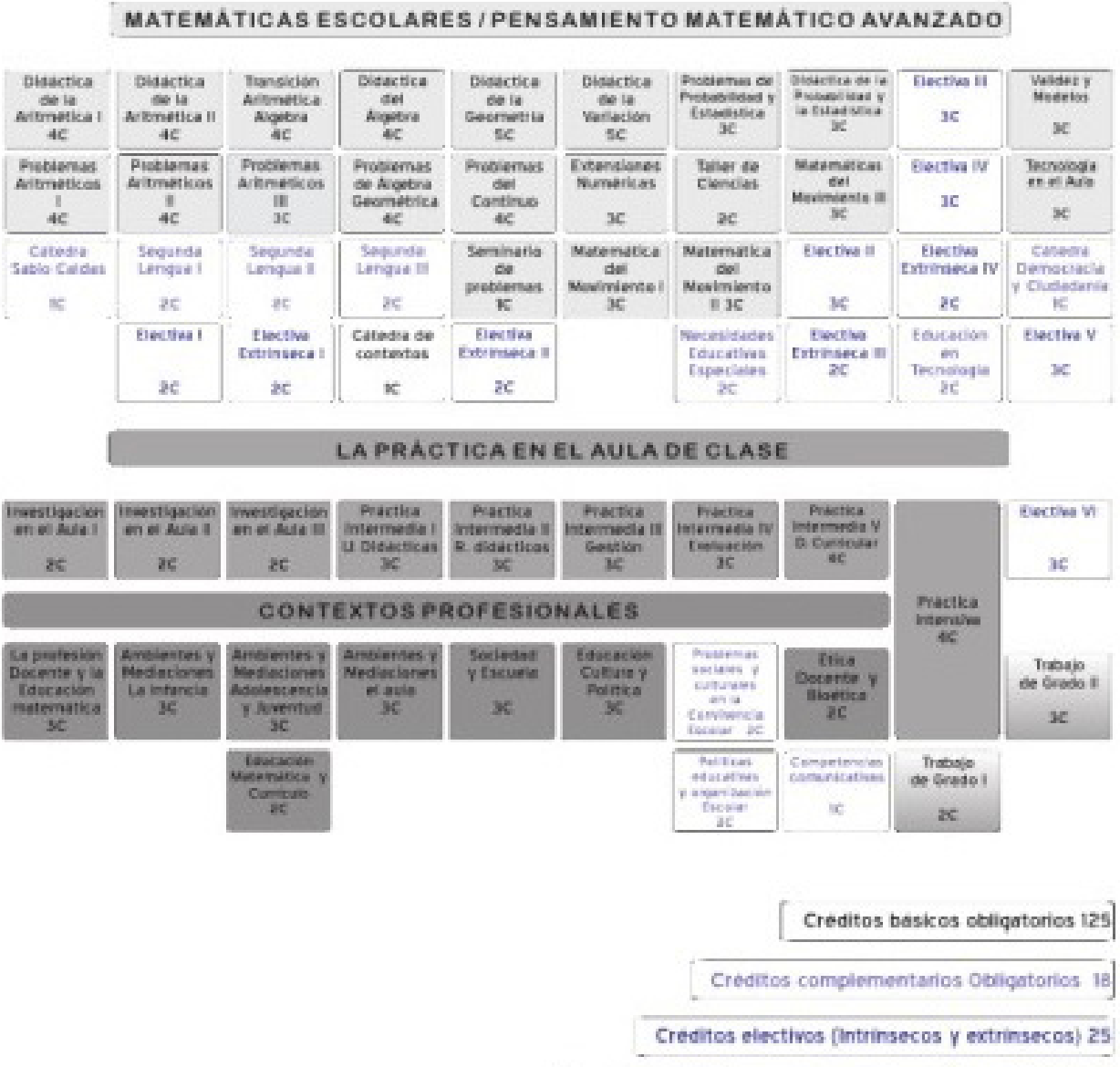

Total de Creditos pars el proyecto curricular LEBEM 168

Fuente: Elaboración propia 
que consideramos pertinente incluir dentro del plan de estudios una asignatura o electiva en etnomatemáticas.

Desde nuestra experiencia como estudiantes de la LEBEM solo hemos tenido dos acercamientos a la etnomatemática, el primero en cuarto semestre en la cátedra de estudios afrocolombianos dada a toda la facultad y la segunda en la asignatura educación matemática y currículo en donde se hizo la elaboración de un trabajo sobre políticas de etnoeducacion, lo que nos lleva a reconocer que a pesar de que se declare el país como plurietnico y multicultural, hace falta que se fortalezca la preparación de docentes para que sean capaces de atender la diversidad presente en el aula.

\section{Conclusiones.}

Al revisar el plan de estudios nos damos cuenta que si se toca el tema de etnomatemáticas en el proyecto es por parte de los docentes, pero de acuerdo a nuestra experiencia como estudiantes de la LEBEM esta iniciativa no se muestra en todos los docentes, por este motivo se puede decir que el tema de etnomatemáticas no es muy conocido y de mucho interés por la comunidad académica.

Así que nosotros pensamos que ya que el plan de estudias está dividido en cuatro ejes: práctica, problemas, didáctica y contextos profesionales, el estudio de las etnomatemáticas debería tocarse en los cuatro ejes o por lo menos en el de contextos profesionales dado que en este se trabajan los aspectos históricos, políticas, psicológicos, culturales, de la educación y la comunidad educativa, o por lo menos crear una electiva intrínseca que trate este tema; hace un unos años existió una llamada etnomatemática, la cual la dictaba el profesor Aldo Iván Parra, pero al este irse el profesor de la universidad, la electiva se cancelo, U otra opción podría ser realizar en una práctica en una zona rural y en colegios o en lugares que se prestan para el desarrollo de las etnomatemáticas (esto sin desconocer que cualquier aula de clase es una aula de diversidad en donde esta presenta le etnoeducacion y por supuesto la etnomatematica)

Como iniciativa actualmente hay una semillero de investigación sobre este tema, el cual se llama "semillero etnomatemática Udistrital" fue creado por la iniciativa de algunos estudiantes del proyecto que están cursando el pregrado o ya están el maestría principalmente.

\section{Referencias}

Blanco, H (2008). Entrevista al profesor Ubiratan D'Ambrosio. Revista Latinoamericana de Etnomatemática, 1(1). 21-25

Castillo, C, (2008). Etnoeducación y políticas educativas en Colombia: la fragmentación de los derechos. Revista Educación y Pedagogía, vol. XX, núm. 52.

Congreso de la república de Colombia. Ley 70 de 1993 (27 de agosto), por la cual se desarrolla el artículo transitorio 55 de la constitución política. Bogotá DC. Colombia.

Congreso de la república de Colombia. Ley 0115 de 1994, (08 de febrero), ley general de educación. Bogotá DC. Colombia.

Constitución política de Colombia, (1991).editorial: Atenea.

Pacheco O, (martes 11 de septiembre de 2007), Introducción a la etnomatemática, disponible en: http:estudiostransculturales. 